\title{
Sensing System for Social Welfare \\ - For easy-to-use interface -
}

\author{
Seiichi Serikawa, \\ ${ }^{a}$ Kyushu Institute of Technology, 1-1 Sensui, Tobata, Kitakyushu, Fukuoka, 804-8550, Japan \\ *Corresponding Author: serikawa@else.kyutech.ac.jp
}

\begin{abstract}
It is often difficult for elderly person to operate the complex wireless remote controllers. This paper introduces our researches to solve the problem. The researches are mainly divided into two aspects; (1) the generalization and customization, and (2) the utilization of the natural human actions. As for (1), touch panel button is introduced. It consists of thin film. That can be bent, cut, and paste anywhere. By applying the film as the touch button, we can generalize it. It can be easily pasted anywhere. In addition, we can cut it as we like. Next, the paper on which buttons are drawn is used as remote controller. The buttons drawn on paper are correctly practiced as remote controller in this study. In addition, a user can make his/her own remote controller by drawing the favorite buttons. As for (2), our researches using the tooth-touch sound and the eyelid motion have been shown. These have the simpler algorithms and the simpler hardware components than those of the conventional researches.
\end{abstract}

Keywords: sensing system, social welfare, user interface.

\section{Introduction}

In Japan, the rate of elderly person is increasing, but that of young person is decreasing ${ }^{(1)}$. The number of elderly people is larger than that of young people. That is serious problem. It will be difficult for young people to care for the elderly in future. Thus, the research of support for elderly is important. There are many healthy elderly people. Some parts of their function are just a little low. If we support the declining function, such persons can self-reliant. So, the study of sensing system is useful to make support system. It can support weak function instead of human's declining sense. Recently, sensing technology has been progressing rapidly. Sensors are used in place of five senses of human. In elderly support systems, the sensor unit is equivalent to the input part of user interface. As the input-interface ${ }^{(2)}$, it is more important to use easily and recognize flexibly than to measure with high sensitivity. For them, a computer is needed. That is the technology of sensor agent ${ }^{(3)}$. A user interface constructed by cheap devices is also needed to spread it to the social life. For example, ubiquitous sensor networks using in the social life use many sensor nodes in the rather wide area. K.-Y. Choia et al. have monitored environmental pollutants in air using gas sensors ${ }^{(4)}$. In such application domains which use many sensors, each node has to be cheap in order to reduce the system cost.

This paper pays attention to the wireless remote controllers using the sensing systems, which exist around us ubiquitously but recently are becoming complex and various. Especially, the handicapped people or the elderly people are hard to operate the complex wireless remote controllers. This paper introduces our researches tackling these problems. The researches are mainly divided into two aspects. One includes the generalization and customization, and another has the utilization of the natural human actions. The first one tries to cope with both the generalization and the customization which are mutually exclusive. The generalization frees the users from having many different controllers. The customization provides our own controllers as we like. The second one attempts to realize the operations by the natural human actions to provide the easy and intuitive controllers. Some case studies are shown. The further advances of the user interface for the sensing systems will provide more comfortable social life for elderly. 


\section{Generalization and Customization of Touch Switch}

There are many kinds of wireless remote controllers for example TV, DVD player, game console, air conditioner, and so on. As functionality of the controllers is becoming complex more and more, the number of buttons is increasing. At the same time, the size of buttons is also becoming smaller and smaller.

Thus, the people, especially the handicapped or elderly one, would be hard to use such remote controllers. Some makers provide an easy controller whose functionality is limited and whose button size is large. However, many buttons can not be placed on a controller, because the size button is large in spite of the limitation of controller size. Thus, the buttons which are frequently used are not always placed on the controller. It is difficult to provide a simple general controller covering all users. From the viewpoint of improving the social life, it is worth developing a controller which is simple and can be customizable for various users.

\subsection{Touch Panel Type of Remote Controller}

To tackle these subjects, we introduce touch panel type of remote controller. The principle to use the touch panel as button is not mentioned here. For the detail, see Ref. (5). That is quite different from conventional touch panel. The material of touch part is thin metal film. It is easy to bend and cut, and can be pasted anywhere. Fig. 1 shows the examples. Fig. 1(a) is the flat type touch, (b) is the arm band type, and (c) is the flower and leaf types. In these examples, each type has 6 touch-buttons. The buttons are assigned to the command "TV on/off", "channel Up", "channel Down", "volume Up", "volume Down", and "change input signal", respectively. The detecting part is connected to remote control. In all cases of Figs. (a), (b) and (c), the touch-button is correctly selected by touching it with finger, and the corresponding remote control code is sent to TV. TV works well by the code. In this case, the number of buttons is set to be 6 , but it is easily changed. In addition, as understood from Fig. 5, we can arbitrary change the shape of button.

By applying the film as the touch button of the remote controller, we can generalize it. It can be easily pasted anywhere. In addition, we can cut it as we like. That means we can decide the shape of the switch by ourselves. Thus, this proposal realizes the generalization and customization into the remote controller.

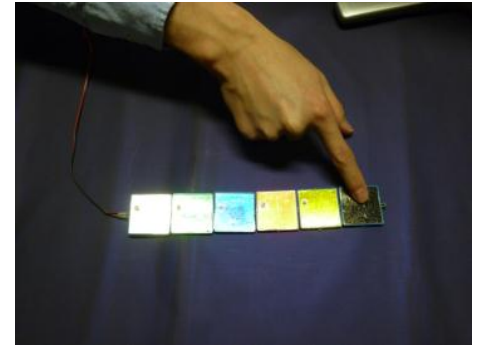

(a) Flat type touch panel.

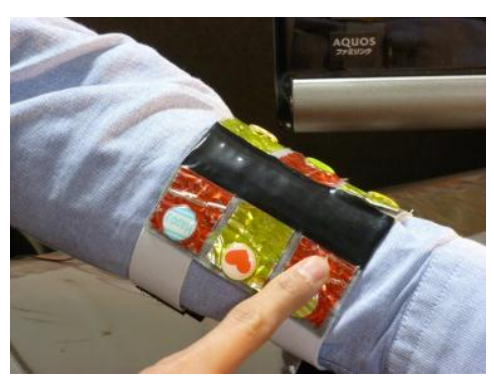

(b) Arm-band type touch panel.

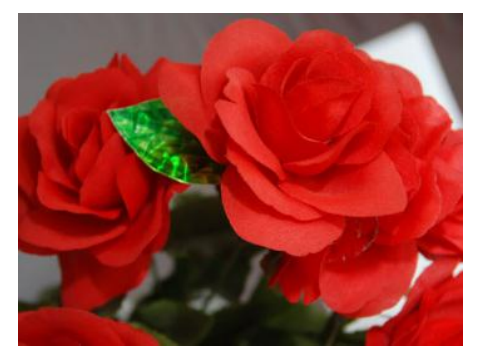

(c) Flower and leaf types touch panel.

Fig. 1. Examples of proposed touch panel. controller.

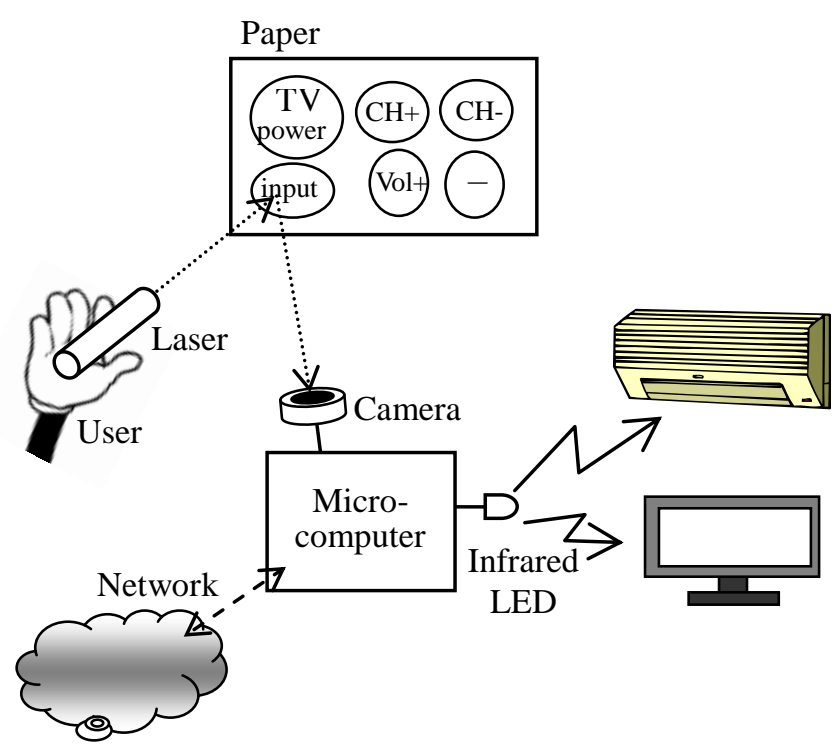

Fig. 2. System Concept of paper type controller. 


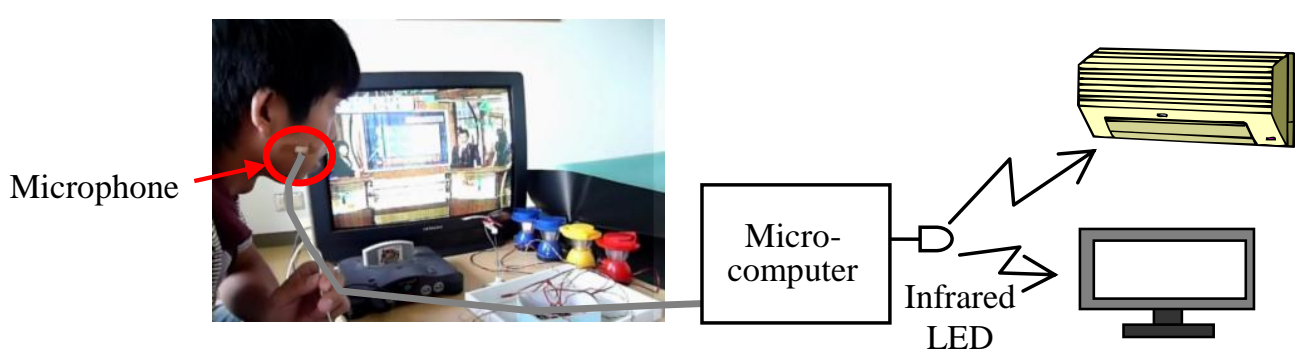

Fig. 3. Remote Control System Using Tooth-touch Sound.

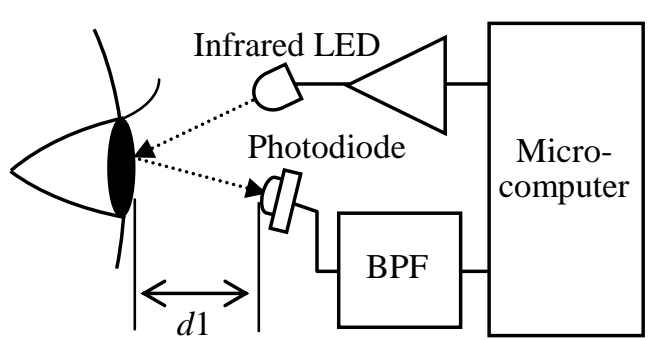

(a) Eye opened

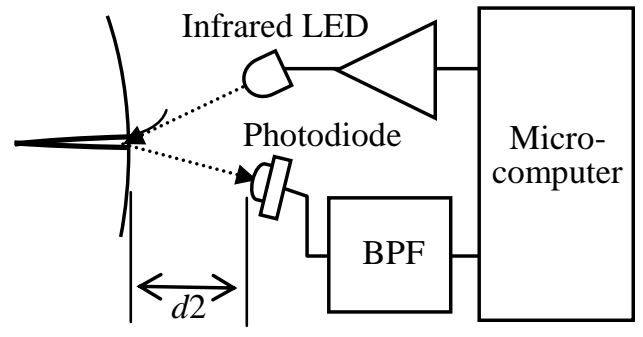

$(d 1>d 2)$

Fig. 4. Idea of Using Eyelid Motion.

\subsection{Paper Type of Remote Controller}

As one of the other user interface, we have proposed a remote controlling system as shown in Fig. $2^{(6)}$. In this system, the buttons of the controller are drawn on a paper by the user with a pen. The user can arbitrarily design the size and arrangement of the buttons. The paper, on which buttons are drawn, can be set onto a preferable place like table, desk and wall. How to use the controller is simple. The user just points out a button on paper with laser pointer for 2 seconds. A microcomputer judges the button is selected or not, and it sends the remote control code to home appliances such as TV, DVD and air conditioner.

By applying the paper as the buttons of the remote controller, the buttons are generalized. This is because everybody easily draws buttons on a paper. In addition, we can get paper easily. Thus, this proposal realizes the generalization and customization into the remote controller.

\section{Utilization of Natural Human Action to Touch Button}

Sometimes a handicapped person would not be able to use remote controller well with his hand. In order to improve the social life for the handicapped people more, the remote controllers that can be operated by the simple actions are needed. Thus, we have proposed some operating methods utilizing the natural human actions ${ }^{(7),(8)}$. Here we demonstrate two types of remote controllers by natural human actions. They use the tooth-touch sound and the eyelid motion.

\subsection{Tooth-touch Sound}

Tooth-touch sound is one of the biomedical signals which are less physical-load. Thus, the tooth-touch sound is a preferable method for the disabled people to remotely control several devices ${ }^{(9)}$. Previous research has used a bone conduction microphone and a complex signal processing to remove the voice signal and the white noise.

In contrast, we have proposed the system shown in Fig. 3. Since this system consists of a conventional microphone and a simple signal processing, a trivial microprocessor can be used. Consequently, the proposed system can be constructed with low price and can contribute to spread the assisting system using the tooth-touch sound.

To simplify the signal processing, the ultrasonic frequency region of sound gotten by microphone is used instead of audible region. The waveform of the tooth-touch sound is quite different from that of voice sound in ultrasonic frequency region ${ }^{(9)}$. This means they can be distinguished in the region. Thus, without a complex signal processing, our proposal within the ultrasonic region can extract the tooth-touch sound easily. By this principle, we can utilize tooth sound easily and correctly as remote controller. 


\subsection{Eyelid Motion ${ }^{(10)}$}

The human routinely and unconsciously opens and closes eyes. Thus, this action is a promising candidate of the biomedical signals to control several devices without physical-load for elderly or disabled persons remotely.

Fig.4 shows our basic idea of utilizing the eyelid motion with a simple hardware and a simple signal processing. The infrared LED irradiates the eye or the eyelid. The photodiode gets the infrared light reflected from eye of eyelid. The output from BPF is the voltage which is proportional to light intensity. When the eye is closed, the distance $d 1$ between the reflecting point and the photodiode is closer than when the eye is opened due to the thickness of the eyelid. The experimental result has shown that the voltage difference which can recognize the eye's status (closed/opened) is shown even if the thickness of the eyelid is commonly small.

Since the output voltage changes by the distance between photodiode and human's eye, it depends on person's setting position. Thus, we can not easily distinguish the state of closed eye from opened eye by a fixed threshold. We have to change the threshold depending on human setting position, but it is difficult. For the improvement, the differential waveform is used. For the detail, see Ref. (10).

\section{Conclusions}

It is often difficult for elderly person to operate the complex wireless remote controllers. This paper introduces our researches to solve the problem. The researches are mainly divided into two aspects; (1) the generalization and customization, and (2) the utilization of the natural human actions.

For the first one, touch panel button is introduced. It consists of thin film. That can be bent, cut, and paste anywhere. By applying the film as the touch button, we can generalize it. It can be easily pasted anywhere. In addition, we can cut it as we like. Thus, the proposed methods realize the generalization and customization into the remote controller. Next, the paper on which buttons are drawn is used as remote controller. Buttons drawn on paper are correctly practiced as remote controller in this study. In addition, a user can make his/her own remote controller by drawing the favorite buttons. The second one has realized the operations by the natural human actions to provide the easy and intuitive controllers. Our researches using the tooth-touch sound and the eyelid motion have been shown. These have the simpler algorithms and the simpler hardware components than those of the conventional researches.

The development of user interface using the sensing systems will provide more comfortable social life.

\section{References}

(1) 2012 World Population Data Sheet, Population reference bureau, pp. 1-20, www.prb.org, 2012.

(2) Rehabilitation engineering, K. Teshima, T. Aikawa, et al, Corona-sha, 2009, in Japanese.

(3) Sensor agent, Sensor agent research committee, Kaibundo, 2003, in Japanese.

(4) K.-Y. Choia, J.-S. Parka, and K.-B. Parka, "Low Power Micro-gas Sensors Using Mixed SnO2 Nanoparticles and MWCNTs to Detect NO2, NH3, and Xylene Gases for Ubiquitous Sensor Network Applications," Sensors and Actuators B: Chemical, Vol. 150, No. 1, pp. 65-72, 2010.

(5) Seiichi Serikawa and Lifeng Zhang, "Proposal of a touch panel switch with the function of bending and addition - one of a new user interface", 2011 2nd International Conference on Instrumentation, Communications, Information Technology, and Biomedical Engineering (ICICI-BME), pp. 8-13, 2011.

(6) Y. Kitazono, K. Ishida, L. Zhang, and S. Serikawa, "Proposal of easily operated remote control using a WEB camera", Proc. of Joint4th International Conference on Soft Computing and Intelligent Systems and9th International Symposium on Advanced Intelligent Systems, pp. 1100-1104, 2008.

(7) D. Baba, S. Nakashima and S. Serikawa, "Proposal of remote control using tooth-touch sound", Proc. of 2008 Joint Conference of Electrical and Electronics Engineers in Kyushu, p. 10-1P-01, 2008, in Japanese.

(8) K. Miyata, Y. Shin, R. Beppu, Y. Kitazono, S. Yang, and S. Serikawa, "Proposal of Wearable User Interface using Movement of Human's Neck”, Proc. of 2009 Joint Conference of Electrical and Electronics Engineers in Kyushu, p. 01-2P-13, 2009, in Japanese.

(9) Akira Yamawaki and Seiichi Serikawa, "A lightweight sensing method of tooth-touch sound for disabled person using remote controller", Journal of Artificial Life and Robotics, Vol. 17, 2012.

(10) Seiichi Serikawa and Akira Yamawaki, "Measurement 
in Social Life - Toward More Efficient User Interface",

Applied Mechanics and Materials, Vol. 103, pp.

592-599, 2011. 